\title{
NOISE REDUCTION METHOD FOR OCT IMAGES BASED ON EMPIRICAL MODE DECOMPOSITION
}

\author{
OLEG O. MYAKININ*,*, DMITRY V. KORNILIN*, \\ IVAN A. BRATCHENKO*, VALERIY P. ZAKHAROV* \\ and ALEXANDER G. KHRAMOV ${ }^{\dagger}$ \\ *Photonics Laboratory \\ Samara State Aerospace University \\ Moskovskoe shosse 34, Samara, Russia 443086, Russia \\ ${ }^{\dagger}$ Laboratory of Laser Measurements \\ Image Processing System Institute of Russian Academy of Science \\ Molodogvardeyskaya Street 151, Samara 443001, Russia \\ *myakole@gmail.com
}

Received 6 November 2012

Accepted 30 December 2012

Published 18 March 2013

\begin{abstract}
In this paper, the new method for OCT images denoizing based on empirical mode decomposition (EMD) is proposed. The noise reduction is a very important process for following operations to analyze and recognition of tissue structure. Our method does not require any additional operations and hardware modifications. The basics of proposed method is described. Quality improvement of noise suppression on example of edge-detection procedure using the classical Canny's algorithm without any additional pre- and post-processing operations is demonstrated. Improvement of rawsegmentation in the automatic diagnostic process between a tissue and a mesh implant is shown.
\end{abstract}

Keywords: Optical coherence tomography; empirical mode decomposition; denoizing; thresholding.

\section{Introduction}

Optical coherence tomography (OCT) is a rapidly developing imaging technology which allows noninvasive cross-sectional imaging of weakly scattering samples with high sensitivity. It has been successfully applied as an imaging tool in medicine. ${ }^{1}$ SD-OCT (OCT with spectral domain detection) technology can reach imaging speeds up to several hundred thousand lines per second.
Speckle pattern (as in every coherent imaging technology) significantly reduces axial and lateral resolution in OCT-setups. Most techniques for reduction of speckle noise uses classical algorithms of speckle reduction ${ }^{2-6}$ or require a priori information about process. ${ }^{7-9}$ Sometimes these algorithms require hardware (and maybe also software) modification of scanning and imaging system. ${ }^{10}$ This list of existing methods for noise reduction (specially

This is an Open Access article published by World Scientific Publishing Company. It is distributed under the terms of the Creative Commons Attribution 3.0 (CC-BY) License. Further distribution of this work is permitted, provided the original work is properly cited. 
for OCT) could be continued. Sometimes such modifications are undesirable or even impossible.

\section{Two-stage Technique for Noise Suppression of OCT-images}

We propose and demonstrate the new two-stage technique for accurate noise suppression of OCTimages: simple pre-filtering (interframe averaging and linear smoothing) on the first stage and empirical mode decomposition (EMD-filtering) on the second stage. The aim of the first stage is to remove ultrahigh frequency modes from the raw data which as usual does not include any useful information about investigated object. The second stage must reduce remaining noise spikes at high frequencies without affecting the fine details of the desired signal.

Raw three-dimensional digital OCT signal $I\left(x_{i}\right.$, $\left.y_{j}, z_{k}\right)$ can be represented as a set of depthward cross-section C-scan images:

$$
I_{k_{0}}\left(x_{i}, y_{j}\right)=\left.I\left(x_{i}, y_{j}, z_{k}\right)\right|_{k=k_{0}} .
$$

As OCT longitudinal resolution is about $5 \mu \mathrm{m}$ each neighboring images cannot differ greatly in structure details. As such first stage of denoizing algorithm may be realized as interframe averaging of $K$ adjacent C-scans:

$$
\hat{I}_{k}\left(x_{i}, y_{j}\right)=\sum_{l=k-r}^{k+r} I_{l}\left(x_{i}, y_{j}\right), \quad \forall(i, j, k), r=\frac{K-1}{2},
$$

with subsequent $3 \times 3$ spatial average linear smoothing (convolution) of each frame:

$$
\tilde{I}_{k}\left(x_{i}, y_{j}\right)=\hat{I}_{l}\left(x_{i}, y_{j}\right)^{* *} h, \quad \forall k, h=\left[\begin{array}{lll}
1 & 1 & 1 \\
1 & 1 & 1 \\
1 & 1 & 1
\end{array}\right] .
$$

First stage pre-filtered OCT C-scan with $K=9$ is shown on Fig. 2(b). The choice of parameter $K$ is individual and depends on images dynamics.

The second stage EMD-filtering is based on onedimensional algorithm of spatial-frequency anal$y \operatorname{sis}^{11}$ with sequential filtering of high frequencies. EMD-procedure is applied for each row (or each column) of the C-scan [see Eq. (1)]. As a result, the original signal is divided into a sum of $N$ quasiorthogonal quasi-periodic intrinsic mode functions
(IMFs) $c_{n}(I)$ and remainder $r(I)$ :

$$
\operatorname{EMD}\left(\tilde{I}_{k}\right)=\sum_{n=1}^{N} c_{n}\left(\tilde{I}_{k}\right)+r\left(\tilde{I}_{k}\right)
$$

IMFs satisfy the following two conditions: (1) in the whole data set, the number of extremes and the number of zero crossings must either be equal or differ at most by one; (2) at any point, the mean value of the envelope curves defined by the local maxima and the local minima is zero.

We propose the following algorithm:

(1) each row (or each column) of the image $\tilde{I}_{k}\left(x_{i}\right.$, $\left.y_{j}\right)$ is subjected to EMD;

(2) over the first few IMFs (in current study $i=1,2)$ the thresholding is performed.

The EMD algorithm for signal $x(t)$ :

(1) Mark the local maxima and minima of signal $x(t)$ and interpolate extreme points by splines to obtain upper and lower envelopes.

(2) Obtain the mean of two envelope curves, $m_{10}(t)$.

(3) Obtain $h_{10}(t)=x(t)-m_{10}(t)$ and check the number of extremes and the number of zero crossings to be equal or differ at most by one. Control all the local maxima to be positive and all local minima to be negative.

(4) If not, repeat the sifting process and obtain $h_{11}$ $(t)=h_{10}(t)-m_{11}(t)\left(m_{11}(t)\right.$ is the mean of the two envelopes of $h_{10}(t)$, see stages 1 and 2 of current algorithm) and repeat:

$$
h_{1 k}(t)=h_{1(k-1)}(t)-m_{1 k}(t) .
$$

(5) If $h_{1 k}(t)$ is an IMF (see two conditions above), then define $c_{1}(t)=h_{1 k}(t)$. Now, we may obtain the first residual as $r_{1}(t)=x(t)-c_{1}(t)$.

(6) Treat $r_{1}(t)$ as a new data set, and perform the sifting process to obtain $c_{2}(t)$.

(7) Continuing the sifting process we obtain

$$
\begin{aligned}
r_{2}(t) & =r_{1}(t)-c_{2}(t), \ldots, r(t)=r_{N}(t) \\
& =r_{N-1}(t)-c_{N}(t) .
\end{aligned}
$$

For our algorithm it is enough to use two IMFs, e.g., $N=2$.

Finally, the original signal is decomposed in IMFs terms. 

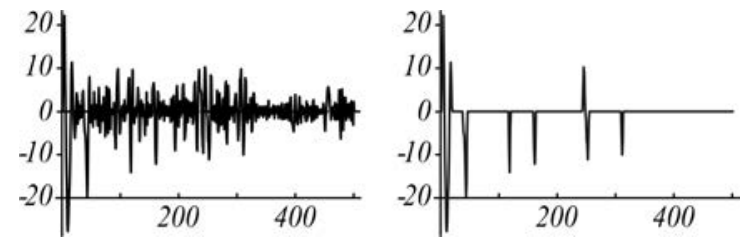

(a)
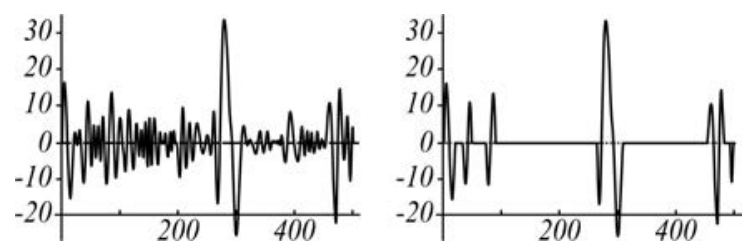

(b)

Fig. 1. Demo of proposed threshold technique: (a) IMF $c_{1}(j)$ and $(\mathrm{b}) c_{2}(j)$ before (left) and after (right) thresholding $(T=10)$.

The authors of Ref. 12 propose to reject first modes for further reduction of the noise. But such procedure leads to the loss of small-size image details. Taking into account that the signal
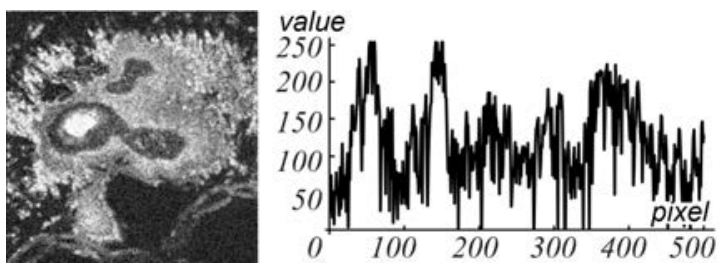

(a)
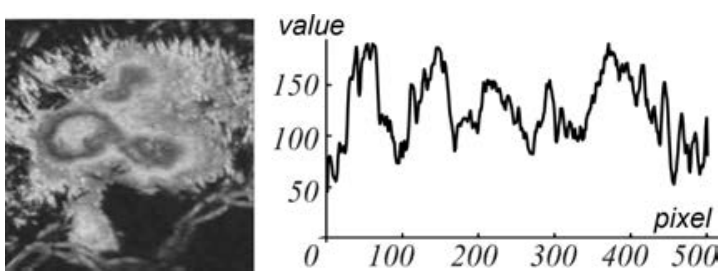

(b)
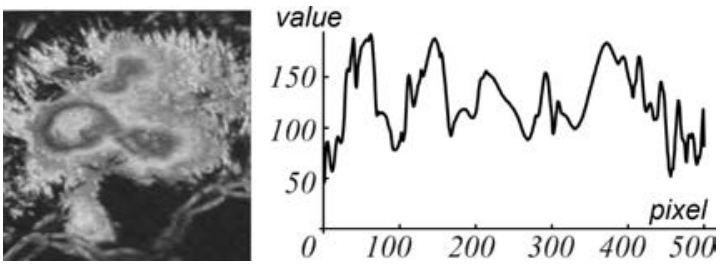

(c)

Fig. 2. C-scans $(500 \times 500 \mathrm{px})$ of SD OCT and its middle $y$-line of (a) raw (b) pre-filtered $(K=9)$; (c) EMD-filtered $(T=10)$ image of implant under the layer of mouse skin.

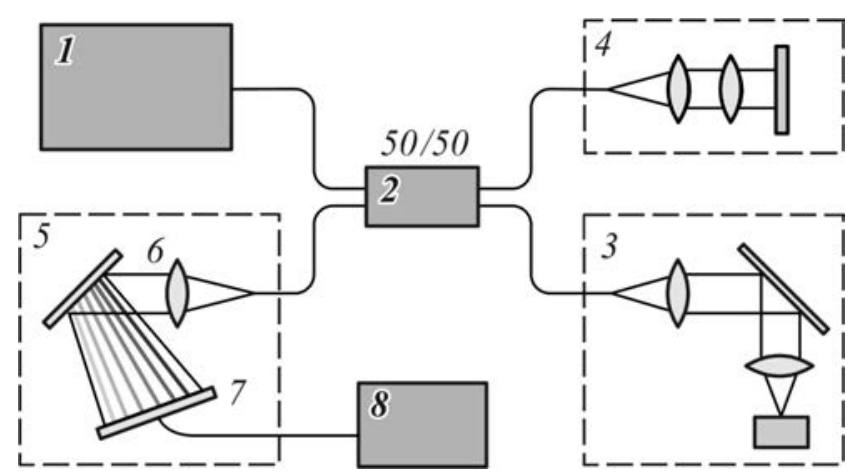

Fig. 3. Spectral domain OCT scheme: 1 - broadband source, 2 - 50/50 beamsplitter, 3 - sample arm, 4 - reference arm, 5- spectrometer with grating 6 and CCD camera 7, 8 - computer with IMAQ.

amplitude is much larger than the noise amplitude we perform thresholding over the first two IMFs, which replace by zero all their spatial half-periods with amplitude less than some pre-assigned threshold $T$ (see Fig. 1). The results of EMD-filtering is shown on Fig. 2(c). The figure shows that the noise was reduced and the small details were saved.

The OCT system (see Fig. 3) comprises of a broadband superluminescent laser diode $(840 \pm$ $25 \mathrm{~nm}$ wavelength range, $20 \mathrm{~mW}$ output power) at the source end, Michelson interferometer with 50/ 50 split ratio to the sample and reference arms and a spectrometer at the detector end. The spectrometer comprises of a diffraction grating (1200 grooves/ $\mathrm{mm}$ ) and a CCD line scan camera (2048 pixel resolution, $29.3 \mathrm{kHz}$ line rate). The interference signal from the sample and the reference arms of the Michelson interferometer is detected by the spectrometer and digitized by an image acquisition card (NI-IMAQ PCI-1428). Depth profile (A-line) is obtained by converting the interference signal detected by the IMAQ into linear $k$-space.

\section{Method of Testing}

For comparative tests we used the following technique:

(1) we took image fragment $300 \times 200$ from leftbottom edge of Fig. 2(a) and made a processing using a few of standard denoizing methods and our proposed EMD-method (see Fig. 4);

(2) we used Canny's edges detector ${ }^{13}$ as test of quality and accuracy for developed EMDmethod (see Fig. 5). 


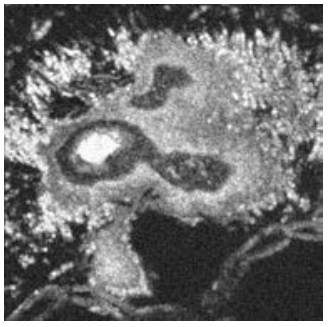

(a)

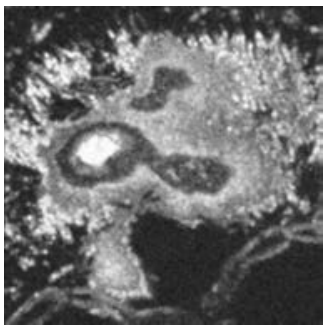

(e)

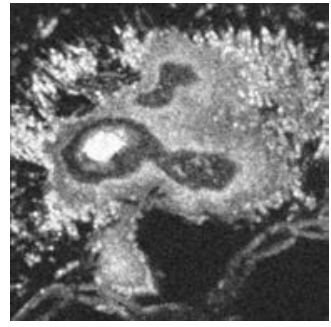

(b)

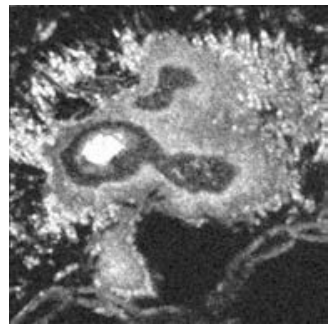

(f)

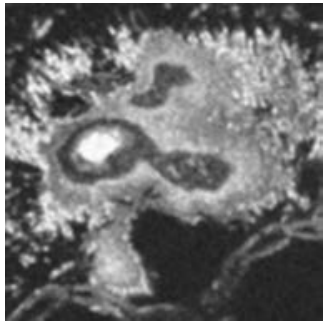

(c)

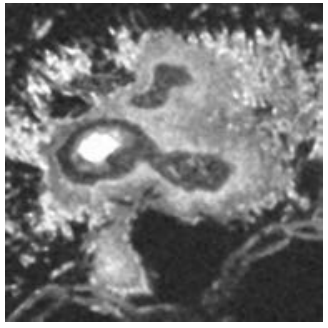

$(\mathrm{g})$

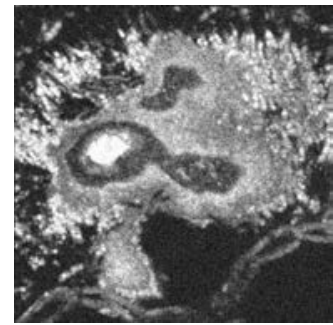

(d)

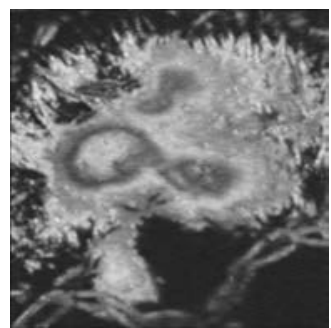

(h)

Fig. 4. Noise reduction for the image of implant under the layer of mouse skin using the different methods.

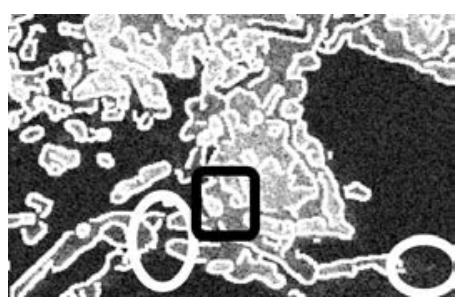

(a)

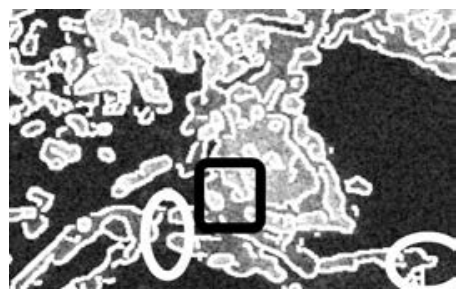

(d)

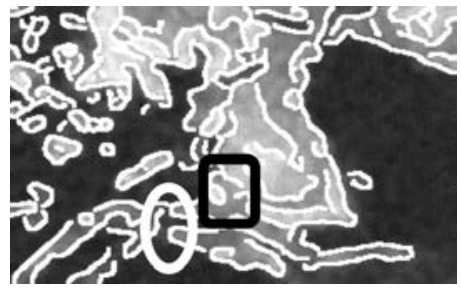

(g)

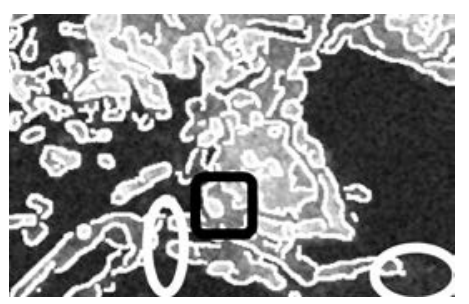

(b)

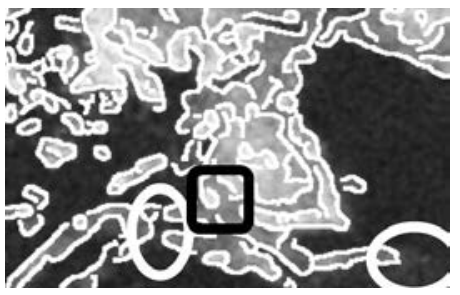

(e)

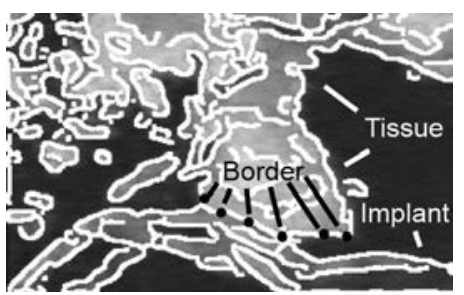

(h)

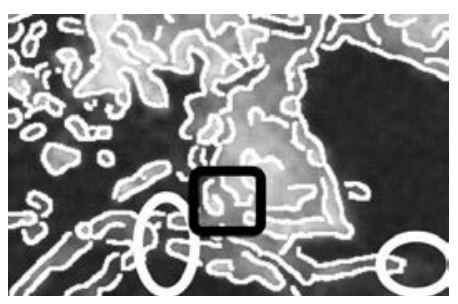

(c)

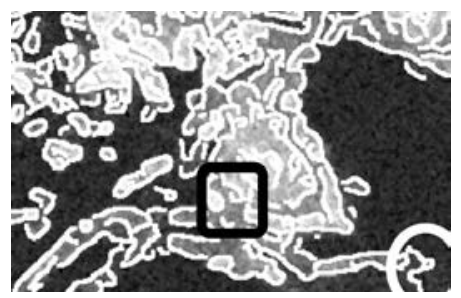

(f)

( Boundary artefacts of implant Boundary artefacts between tissue and implant

Fig. 5. Edge detection for Fig. 4 using Canny algorithm. 
The list of standard denoizing methods for original image fragment [see Fig. 4(a)]:

(1) standard smooth linear filter with window size $3 \times 3$ [see Fig. 4(b)] and $7 \times 7$ [see Fig. 4(c)];

(2) Gaussian smooth filter with core radius 1 [see Fig. 4(d)] and 3 [see Fig. 4(e)];

(3) Median filter with window size $3 \times 3$ [see Fig. $4(\mathrm{f})]$ and $7 \times 7$ [see Fig. $4(\mathrm{~g})]$.

Figure $4(\mathrm{~h})$ is the result of denoizing with using EMD-method.

\section{Discussion}

For Canny detector we did not use any additional pre- or post-processing filters. However, we improved the result using EMD-method: the border between tissue and implant have no artefacts [see Fig. 5(h)] unlike the results of other methods [see Figs. 5(a)$5(\mathrm{~g})]$ of denoizing. Therefore, we obtained image with more accurate boundaries between tissue and implant and as a result more accurate implant's fibers detecting (i.e., boundaries have fewer breaks and other artifacts).

On the other hand, EMD-method has a very important drawback — a very low performance. This method requires multiple interpolation process on irregular two-dimensional lattice which affects the performance.

\section{Acknowledgments}

This research was supported by the Federal Target Program "Research and development on priority directions of Russian scientific-technological complex for 2007-2013".

\section{References}

1. D. Huang, E. A. Swanson, C. P. Lin, J. S. Schuman, W. G. Stinson, W. Chang, M. R. Hee, T. Flotte, K. Gregory, C. A. Puliafito, J. G. Fujimoto, "Optical coherence tomography," Science $\mathbf{2 5 4}$ (5035), 1178-1181 (1991).

2. J. Lee, "Digital image enhancement and noise filtering by use of local statistics," IEEE Trans Pattern Anal. Comput. Intell. 2, 165-168 (1980).
3. D. Kuan, A. Sawchuk, T. Strand, P. Chave, "Adaptive noise smoothing filter for images with signal-dependent noise," IEEE Trans Pattern Anal. Comput. Intell. 7, 165-177 (1985).

4. V. Frost, J. Stiles, K. Shanmugan, J. Holtzman, "A model for radar images and its applications to adaptive digital filtering of multiplicative noise," IEEE Trans Pattern Anal. Comput. Intell. 4, 157-166 (1982).

5. J. Verhoeven, J. Thijssen, "Improvement of lesion detectability by speckle reduction filtering: A quantitative study," Ultrason Imag. 15, 181-204 (1993).

6. J. Rogowska, M. Brezinski, "Evaluation of the adaptive speckle suppression filter for coronal optical coherence tomography imaging," IEEE Trans. Med. Imaging 19, 1261-1266 (2000).

7. S. Bhat, I. V. Larina, K. V. Larin, M. E. Dickinson, M. Liebling, "Multiple-cardiac-cycle noise reduction in dynamic optical coherence tomography of the embryonic heart and vasculature," Opt. Lett. 34, 3704-3706 (2009).

8. J. B. Yoo, I. V. Larina, K. V. Larin, M. E. Dickinson, M. Liebling, "Increasing the field-of-view of dynamic cardiac OCT via post-acquisition mosaicing without affecting frame-rate or spatial resolution," Biomed. Opt. Express 2, 2614-2622 (2011).

9. I. V. Larina, K. V. Larin, M. E. Dickinson, M. Liebling, "Sequential Turning Acquisition and Reconstruction (STAR) method for four-dimensional imaging of cyclically moving structures," Biomed. Opt. Express 3, 650-660 (2012).

10. M. Szkulmowski, I. Gorczynska, D. Szlag, M. Sylwestrzak, A. Kowalczyk, M. Wojtkowski, "Efficient reduction of speckle noise in optical coherence tomography," Opt. Express 20(2), 1337-1359 (2012).

11. N. E. Huang, Z. Shen, S. R. Long, M. C. Wu, H. H. Shih, Q. Zheng, N.-C. Yen, C. C. Tung, H. H. Liu, "The empirical mode decomposition and the Hilbert spectrum for nonlinear and non-stationary time series analysis," Proc. R. Soc. Lond. A. 454, 903-995 (1998).

12. M. B. Bernini, A. Federico, G. H. Kaufmann, "Normalization of fringe patterns using the bidimensional empirical mode decomposition and the Hilbert transform," Appl. Opt. 48, 6862-6869 (2009).

13. J. A. Canny, "Computational approach to edge detection," IEEE Trans. Pattern Anal. Mach. Intell. 8(6), 679-698 (1986). 